\title{
MAPEAMENTO DO ÍNDICE DE VEGETAÇÃO DA DIFERENÇA NORMALIZADA EM LAVOURA DE ALGODÃO ${ }^{1}$
}

\author{
Anamari Viegas de Araujo Motomiya ${ }^{2}$, José Paulo Molin³, Wagner Rogerio Motomiya4a , Fábio Henrique Rojo Baio ${ }^{5}$
}

\begin{abstract}
MAPPING OF THE NORMALIZED DIFFERENCE VEGETATION INDEX IN COTTON FIELD

The remote sensing data obtained at field level can provide detailed information about the variability of biophysical parameters related to yield over large areas, and present potential for monitoring these parameters throughout the crop development cycle. This study aimed to map the spatial variability of the Normalized Difference Vegetation Index (NDVI) and its components in two commercial cotton (Gossipium hirsutum L.) fields, by using an active optical sensor at the ground level. Data were collected with the aid of an optical sensor installed in a self-propelled agricultural sprayer. A GPS receiver was connected to the sensor, in order to obtain the coordinates of the sampling points. The readings were performed in rows spaced $21.0 \mathrm{~m}$ apart by the sensor installed on a vehicle, during the spraying operation, and data analyzed by using the classical statistics and geostatistics. Spatial distribution maps of the variables were generated by kriging interpolation. It was observed a higher spatial variability of NDVI and spectral reflectance of vegetation in the region of near infrared (NIR) $(880 \mathrm{~nm})$ and visible infrared $(590 \mathrm{~nm})$ in the crop with higher physiological stress, due to the brown bug [Scaptocoris castanea (Hem.: Cydnidae)] attack, when compared to the healthy one.
\end{abstract}

KEY-WORDS: Gossypium hirsutum L.; geoestatistics; remote sensing; spatial variability; active optical sensor.

\section{INTRODUÇÃO}

Avanços tecnológicos na agropecuária têm mostrado a importância de se medir a variabilidade espacial e temporal de alguns indicadores das condições fitossanitárias que afetam o rendimento

\section{RESUMO}

Os dados de sensoriamento remoto em campo podem fornecer informações detalhadas sobre a variabilidade de parâmetros biofísicos ligados à produtividade em grandes áreas e apresentam potencial para o monitoramento destes parâmetros, ao longo de todo o ciclo de desenvolvimento da cultura. Este trabalho objetivou mapear a variabilidade espacial do índice de vegetação da diferença normalizada (NDVI) e seus componentes, em duas lavouras comerciais de algodão (Gossipium hirsutum L.), utilizando sensor óptico ativo, em nível terrestre. Os dados foram coletados utilizando-se sensor instalado em um pulverizador autopropelido agrícola. Um receptor GPS foi acoplado ao sensor, para a obtenção das coordenadas dos pontos de amostragem. As leituras foram realizadas em faixas espaçadas em 21,0 m, aproveitando-se as passadas do veículo no momento da pulverização de agroquímicos, e os dados submetidos à análise estatística clássica e geoestatística. Mapas de distribuição espacial das variáveis foram elaborados pela interpolação por krigagem. Observou-se maior variabilidade espacial do NDVI e da reflectância espectral da vegetação na região do infravermelho próximo (IVP) $(880 \mathrm{~nm})$ e do visível $(590 \mathrm{~nm})$ na lavoura com maior estresse fisiológico, devido ao ataque do percevejo castanho [Scaptocoris castanea (Hem.: Cydnidae)], em relação à lavoura sadia.

PALAVRAS-CHAVE: Gossypium hirsutum L.; geoestatística; sensoriamento remoto; variabilidade espacial; sensor óptico ativo.

das culturas (Carvalho et al. 2002), como base para administrar as operações de manejo (Mulla \& Schepers 1997, Goel et al. 2003). Isto permite que a aplicação de insumos seja ajustada localmente, em nível adequado (Booltink \& Verhagen 1997, Goel et al. 2003).

1. Trabalho recebido em maio/2011 e aceito para publicação em jan./2012 ( $\mathrm{n}^{\circ}$ registro: PAT 14251).

2. Universidade Federal da Grande Dourados, Faculdade de Ciências Agrárias, Dourados, MS, Brasil. E-mail: anamarimotomiya@ufgd.edu.br.

3. Universidade de São Paulo, Escola Superior de Agricultura Luiz de Queiroz, Departamento de Engenharia de Biossistemas, Piracicaba, SP, Brasil. E-mail: jpmolin@usp.br.

4. Monsanto do Brasil Ltda., Departamento de Regulamentação, Rolândia, PR, Brasil. E-mail: wagner.r.motomiya@monsanto.com.

5. Universidade Federal de Mato Grosso do Sul, Campus Universitário de Chapadão do Sul, Chapadão do Sul, MS, Brasil.

E-mail: fabiobaio@ufms.br. 
Durante as duas últimas décadas, o desenvolvimento na capacidade de aquisição, processamento e interpretação dos dados terrestres, aéreos e de satélites tem tornado possível utilizar as tecnologias do sensoriamento remoto em conjunto com os sistemas de agricultura de precisão. As técnicas ligadas ao Sensoriamento Remoto (SR) surgem como instrumental de elevado potencial, tanto na coleta como no processamento de dados referentes às áreas agrícolas. Os dados de SR, tais como imagens de satélite, fotografias aéreas e radiometria de campo, podem fornecer informações precisas da variabilidade do campo, em grandes áreas, por apresentarem potencial para o monitoramento de parâmetros biofísicos ligados à produtividade, ao longo de todo o ciclo de desenvolvimento da cultura.

A mensuração da reflectância espectral é a abordagem sem contato e não destrutiva mais promissora para a determinação da deficiência de nitrogênio nas culturas (Tumbo et al. 2002). A luz vermelha é absorvida pelos pigmentos verdes (clorofila), em tecidos fotossinteticamente ativos, e, portanto, a proporção refletida varia inversamente à quantidade de biomassa vegetal. No entanto, no campo, a intensidade de luz vermelha refletida vai depender não apenas da proporção absorvida, mas, também, da intensidade incidente, que varia com a localização e hora do dia. Segundo Lawrence et al. (2000), métodos práticos de determinação de biomassa vegetal geralmente levam este fator em conta, medindo, simultaneamente, outro comprimento de onda, que é relativamente pouco afetado pela presença de material verde, expressando o resultado como uma relação entre as duas intensidades. A relação mais comumente utilizada é o índice de vegetação da diferença normalizada, definido como NDVI = (IVP - Vis $) /(I V P+V i s)$, onde IVP é a intensidade de reflectância no infravermelho próximo e Vis a intensidade de reflectância do vermelho no visível. O IVP é escolhido como referência, por se difundir pelas superfícies foliares, como resultado de refrações múltiplas entre os espaços intercelulares e as células hidratadas, sendo pouco afetado pelos pigmentos vegetais.

Muitos pesquisadores já utilizaram dados de SR para estimar parâmetros das culturas, tais como radiação fotossinteticamente ativa e índice de área foliar (Baret \& Guyot 1991), conteúdo de clorofila nas folhas (Tumbo et al. 2002), conteúdo de nitrogênio (Solie et al. 2002, Motomiya et al. 2009) e muitas outras propriedades químicas da vegetação.
A utilização de técnicas geoestatísticas e amostragens em grade podem fornecer uma base confiável para a identificação da variabilidade espacial e mapeamento de atributos físicos (Andrade et al. 2005, Vieira et al. 2007) e químicos do solo (Motomiya et al. 2006, Souza et al. 2007, Faria et al. 2009) e das plantas (Silva et al. 2008). Entretanto, em áreas extensas, o custo das análises pode ser proibitivo. Assim, o sensoriamento remoto, ao nível terrestre, produz dados em grande quantidade, os quais serão submetidos à análise geoestatística e podem ser tornar ferramenta útil no mapeamento de áreas extensas. Assim, obtêm-se estimativas confiáveis da variabilidade de indicadores das condições fitossanitárias e nutricionais da vegetação.

Este trabalho teve como objetivo mapear a variabilidade espacial do NDVI e dos seus componentes em duas lavouras comerciais de algodão, utilizando sensor óptico ativo, em nível terrestre.

\section{MATERIAL E MÉTODOS}

O estudo da variabilidade espacial dos índices de reflectância, na cultura do algodoeiro, foi realizado em dois talhões de lavouras comerciais, em Chapadão do Sul (MS), em 2008.

Os dados espectrais foram coletados com um sensor ativo (Crop Circle ${ }^{\circledR}$ ACS-210, Holland Scientific, Inc. Lincoln, NE), que emite a luz no comprimento de onda do vermelho âmbar a $590 \mathrm{~nm}$ (visível) e do infravermelho próximo a $880 \mathrm{~nm}$, sendo a luz refletida captada pelo detector. A partir da refletância da cultura, o sensor, associado a um coletor de dados e a um programa, ambos dedicados, calcula, automaticamente, o índice de vegetação da diferença normalizada (NDVI). De acordo com o fabricante, a altura de trabalho recomendada para o sensor pode variar de $25,0 \mathrm{~cm}$ a $215,0 \mathrm{~cm}$ de distância ao alvo.

Utilizou-se um receptor GPS (Garmin E-Trex, trabalhando no sistema WGS84, conectado ao sensor via RS232, por um protocolo de comunicação NMEA), acoplado ao sensor ativo, para obter as coordenadas dos pontos de amostragem.

$\mathrm{O}$ sensor foi montado sobre um pulverizador autopropelido Uniport (Máquinas Agrícolas Jacto S. A.), com barra de $21,0 \mathrm{~m}$. As leituras foram feitas no momento da pulverização, com o sensor localizado no centro e à frente do veículo. A altura do sensor ao alvo variou de cerca de $0,5 \mathrm{~m}$ a 1,0 $\mathrm{m}$, em função da irregularidade da altura das plantas. Os dados foram 
coletados com frequência de $1 \mathrm{~Hz}$ e velocidade em torno de 5,0 $\mathrm{m} \mathrm{s}^{-1}\left(18,0 \mathrm{~km} \mathrm{~h}^{-1}\right)$, no espaçamento de $21,0 \mathrm{~m}$ entre passadas, o que gerou 75 e 63 pontos por hectare, nas áreas 1 e 2, respectivamente. Em ambos os locais, o espaçamento entrelinhas foi de $90,0 \mathrm{~cm}$, com densidade desejada de 10 plantas por metro, sendo a semeadura direta sobre a palhada de milheto. Os dados foram gravados diretamente no coletor do próprio sensor.

Aárea 1 não apresentava problemas visíveis no seu desenvolvimento e a área 2 apresentava ataque visível de percevejos [Scaptocoris castanea (Hem.: Cydnidae)]. No momento da coleta dos dados, a área 1 encontrava-se em estádio F1 e a área 2 em F6 (Marur \& Ruano 2001).

Os dados foram submetidos a análise estatística, sendo determinados a média, valores máximos e mínimos, coeficientes de assimetria e curtose, coeficiente de variação (CV) e distribuição de frequência dos dados. A análise de dependência espacial foi realizada através do ajuste de modelos ao semivariograma experimental, de acordo com a teoria das variáveis regionalizadas (TVR), utilizando-se o programa GS+ (Robertson 1998).

Uma vez verificada a dependência espacial, foi realizada a interpolação por krigagem, para estimar valores em locais não amostrados, sem tendência e com variância mínima. Após a interpolação, foram elaborados mapas de distribuição espacial das reflectâncias na faixa do visível, do IVP e do NDVI da cultura do algodoeiro.

\section{RESULTADOS E DISCUSSÃO}

A obtenção dos dados de reflectância dos componentes do NDVI, utilizando sensor ótico ativo sobre veículo, em operações agrícolas, é simples e permite a coleta dos dados sem intervir nas atividades de rotina na lavoura. A densidade amostral pode, ainda, ser alterada, em função da largura das passadas do veículo, ou com a adição de mais sensores ao longo da largura do equipamento.

Na Tabela 1, são apresentados os resultados da análise estatística descritiva do índice de vegetação da diferença normalizada e das reflectâncias, no visível e no infravermelho próximo, das plantas de algodoeiro em duas lavouras com condições distintas: área 1: desenvolvimento normal da cultura; e área 2: lavoura com grandes manchas, devido ao ataque do percevejo castanho.

Em média, os valores do NDVI foram semelhantes, nas duas áreas, inclusive em relação à amplitude entre máximos e mínimos. Entretanto, este fato não deve ser considerado isoladamente, pois a avaliação, na área 1, ocorreu 50 dias após a avaliação da área 2, ou seja, apesar de ambas as áreas se encontrarem na fase de florescimento, a área 1 encontrava-se no estádio F1 (abertura da primeira flor), enquanto a área 2 encontrava-se em F6 (abertura da primeira flor no sexto ramo reprodutivo). Assim, o índice de área foliar, o qual tem influência no NDVI, das duas lavouras foi influenciado pelo estádio de desenvolvimento da cultura.

A distribuição de frequência dos dados não se ajustou à normal e a área 2 apresentou coeficientes de assimetria e curtose mais acentuados, indicando maior variabilidade dos dados. Entretanto, conforme salientaram Isaaks \& Srivastava (1989), mais importante que a normalidade dos dados é que os semivariogramas apresentem patamares bem definidos, para não comprometer as estimativas da krigagem, as quais são baseadas nos valores médios.

Segundo critérios estabelecidos por Wilding \& Drees (1983), o coeficiente de variação foi baixo para o NDVI e Vis e moderado para o IVP, na área 1, e baixos para o NDVI e altos para o IVPe Vis, na área 2.

Tabela 1. Estatística descritiva do NDVI e reflectância no infravermelho próximo (IVP) e no visível (Vis), em lavoura de algodão sadia (área 1, estádio F1) e atacada por percevejo castanho (área 2, estádio F6) (Chapadão do Sul, MS, 2008).

\begin{tabular}{lccccccrrr}
\hline Atributos & Média & Desvio & CV (\%) & Mínimo & Máximo & N observações $^{\text {A Assimetria }}$ & Curtose \\
\hline NDVI & 0,73 & 0,06 & 8,23 & 0,10 & 0,82 & 15.248 & $-2,78$ & 15,07 \\
IVP & 0,52 & 0,12 & 23,08 & 0,14 & 0,97 & 15.247 & 0,21 & $-0,02$ \\
Vis & 0,08 & 0,01 & 12,50 & 0,06 & 0,29 & 15.247 & 6,60 & 183,00 \\
\hline & & & & & Área 1 & & \\
NDVI & 0,73 & 0,09 & 12,33 & 0,11 & 0,82 & 5.048 & $-3,65$ & 16,80 \\
IVP & 0,83 & 0,52 & 62,65 & 0,10 & 3,88 & 5.049 & 1,27 & 1,46 \\
Vis & 0,11 & 0,05 & 45,45 & 0,05 & 0,64 & 5.048 & 1,79 & 4,54 \\
\hline
\end{tabular}


Em condições controladas, Motomiya et al. (2009) observaram que os valores do coeficiente de variação do NDVI foram baixos e apresentaram tendência de diminuição, ao longo do ciclo do algodoeiro.

A reflectância no IVP foi maior na lavoura atacada por percevejos (área 2), a qual também apresentou maior variabilidade. Segundo Moreira (2001), o comportamento da radiação eletromagnética, na região do IVP, é dominado pelas propriedades ópticas da folha, que dependem das estruturas celulares internas. Além disto, há uma contribuição indireta do conteúdo de água da folha. Neste caso, como o ataque de percevejos afeta diretamente as raízes, responsáveis pela absorção tanto de água quanto de nutrientes, a estrutura anatômica do mesófilo foliar pode ter sido alterada, causando a maior reflectância na lavoura atacada do que na lavoura sadia.

Li et al. (2000) observaram que a reflectância aumentou significativamente no IVP e diminuiu no vermelho, com o aumento da irrigação, biomassa e absorção de $\mathrm{N}$ pelo algodoeiro. Como a área 2 estava em estágio mais avançado de desenvolvimento, esta apresentava vegetação mais densa, com menos solo descoberto, o que explica os resultados obtidos. Neste sentido, como afirmaram Ponzoni \& Shimabukuro (2009), quanto maior o índice foliar, menor a reflectância no visível e maior no IVP. Entretanto, esta dinâmica não é linear, pois há um valor de IAF acima do qual não ocorre alteração na reflectância do dossel, tanto para a região do visível (tendo atingido um valor mínimo) quanto para o IVP (tendo atingido uma valor máximo).

$\mathrm{Na}$ faixa do visível, observou-se menor reflectância também na área 1 , indicativo do melhor estado fisiológico da cultura, ou seja, como a reflectância foi menor, maior parte da radiação eletromagnética foi absorvida pelos pigmentos fotossintetizantes no me- sófilo foliar. Isto indica que as plantas apresentavam maior quantidade de pigmentos fotossintetizantes, responsáveis pela coloração verde das folhas.

$\mathrm{Na}$ área 2, ocorreram manchas de solo exposto, devido ao ataque dos percevejos, o que interferiu nas propriedades de reflectância da lavoura. Em plantas doentes, há menor produção de clorofilas "a" e "b" e maior produção de carotenoides, cuja função biológica é proteger contra danos oxidativos, e são geralmente usados no sistema de defesa da planta (Julsing et al. 2006).

Segundo Ma et al. (2001), as técnicas de sensoriamento remoto, em particular a reflectância multiespectral do visível e do infravermelho, podem proporcionar dados para realizar correlações entre a reflectância do dossel da planta e a biomassa existente acima do solo, que podem ser usadas para obtenção de estimativas do rendimento das culturas.

Os resultados da análise geoestatística encontram-se na Tabela 2. Somente semivariogramas experimentais unidirecionais foram ajustados aos dados usando o modelo melhor ajustado, com menor soma do resíduo ao quadrado (SRQ).

$\mathrm{O}$ efeito pepita $\left(\mathrm{C}_{0}\right)$ representa a variância não explicada, ou ao acaso, frequentemente causada por erros de medições ou variações das propriedades que não podem ser detectadas na escala de amostragem (Vieira 2000).

Mendes et al. (2008) consideram que o semivariograma apresenta efeito pepita puro quando não é possível identificar a estrutura da variância e os valores da semivariância se mantêm a um determinado nível, comportando-se de forma mais ou menos constante, independentemente do aumento da distância entre amostras. Assim, pode-se assumir que, além de a distribuição ocorrer completamente ao acaso, há independência entre as amostras e os métodos da esta-

Tabela 2. Resultados da análise geoestatística do NDVI e reflectância no infravermelho próximo (IVP) e no visível (Vis), em lavoura de algodão sadia (área 1) e atacada por percevejo castanho (área 2) (Chapadão do Sul, MS, 2008).

\begin{tabular}{llllllll}
\hline \multicolumn{1}{c}{ Atributos } & Modelo & $\mathrm{C}_{0}$ & $\mathrm{C}_{0}+\mathrm{C}$ & $\mathrm{A}_{0}$ & $\mathrm{GDE}$ & $\mathrm{R}^{2}$ & $\mathrm{SRQ}$ \\
\hline NDVI & Exponencial & 0,0009 & 0,0029 & 20,50 & 68,97 & 0,99 & $1,798 \mathrm{e}-08$ \\
IVP & Exponencial & 0,0016 & 0,0112 & 25,10 & 85,71 & 0,98 & $1,107 \mathrm{e}-06$ \\
Vis & Efeito pepita & & & & & \\
\hline & & & & & & \\
NDVI & Exponencial & 0,0017 & 0,0063 & 4,40 & 73,02 & 0,39 & $2,106 \mathrm{e}-06$ \\
IVP & Exponencial & 0,0001 & 0,2810 & 20,20 & 99,96 & 0,99 & $3,268 \mathrm{e}-04$ \\
Vis & Exponencial & 0,0000 & 0,0030 & 20,30 & 100,00 & 0,99 & $6,467 \mathrm{e}-08$ \\
\hline
\end{tabular}

$\mathrm{C}_{0}$ : efeito pepita; $\mathrm{A}_{0}$ : alcance; GDE: grau de dependência espacial; $\mathrm{R}^{2}$ : coeficiente de determinação do modelo; SRQ: soma do resíduo ao quadrado. 
tística clássica que podem ser aplicados, com a média aritmética representando bem o conjunto de dados. No entanto, isto não significa, necessariamente, que não exista estrutura de variância. Provavelmente, neste caso, a dependência espacial ocorreu em uma escala de distância menor do que a que foi utilizada entre os pontos de amostragem.

Conforme critérios estabelecidos por Cambardella et al. (1994), nas duas áreas, o NDVI apresentou moderado GDE, enquanto, na região do IVP, observou-se forte dependência espacial. Na região do visível, apenas na área 2 houve ajuste ao semivariograma experimental, com os dados apresentando forte dependência espacial. $\mathrm{Na}$ área 1 , ao contrário, os dados da região do visível apresentaram efeito pepita puro.

Dentre os parâmetros do semivariograma, o alcance tem importância por revelar a distância limite da dependência espacial. Além desta distância, as amostras apresentam independência e não são correlacionadas entre si. Desta forma, as interpolações devem ser realizadas entre as amostras localizadas dentro do limite de dependência espacial. Na área 1 , os valores do alcance foram de 20,5 m, para o NDVI, e 25,1 m, para o IVP. O alcance do NDVI, na área 2, foi menor (apenas 4,4 m), indicativo da grande descontinuidade espacial desta variável, a qual foi influenciada pelos danos causados pelo ataque dos percevejos. A reflectância no IVP e visível apresentaram valores de alcance bastante próximos e forte GDE.

Os parâmetros dos semivariogramas ajustados foram usados para a elaboração dos mapas de distribuição espacial das variáveis (Figuras 1 e 2). Nitidamente, percebe-se que a lavoura com aspecto mais sadio apresenta áreas mais extensas, com valores de NDVI semelhantes, enquanto, na lavoura atacada, há incidência maior de manchas, indicativas da grande variabilidade dos dados.

No campo, durante a aquisição de dados, observou-se que as áreas com maior incidência de sintomas de ataque de percevejos castanhos apresentavam coloração mais clara (amarelada), o que pode provocar alterações nos valores do NDVI. Os pontos com valores muito baixos de NDVI referem-se a áreas de solo descoberto (bordas do talhão), devido a falhas no estande dentro da lavoura, ou vegetação subdesenvolvida. As áreas com vegetação com bom desenvolvimento apresentaram maiores valores de NDVI. As leituras resultaram em valores mais baixos,
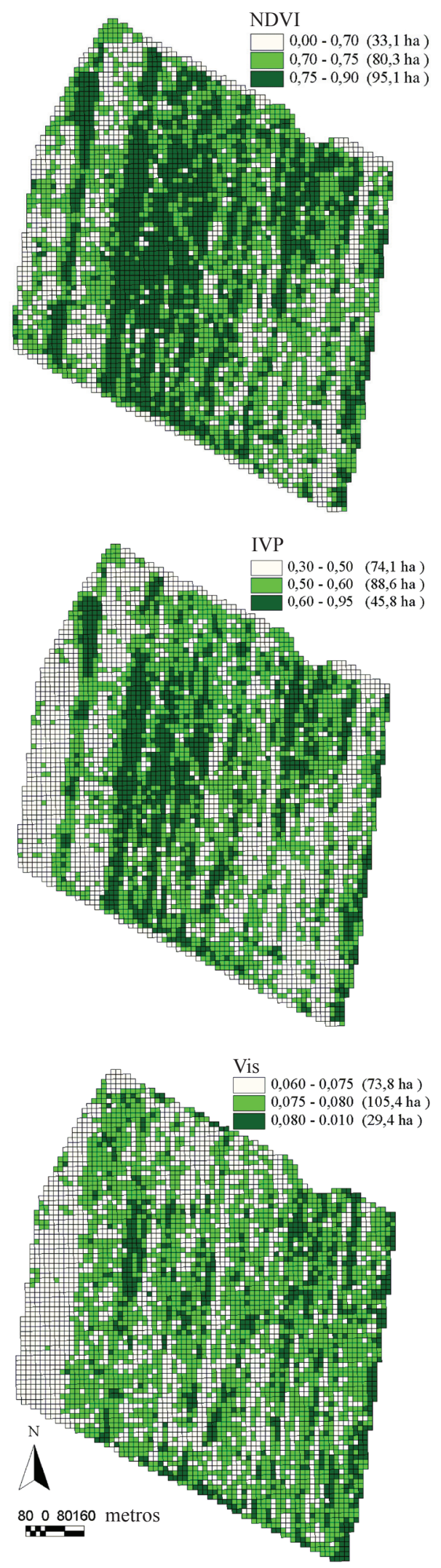

Figura 1. Mapas da distribuição espacial do NDVI, reflectância no infravermelho próximo (IVP) e no visível (Vis), na área 1 (Talhão 1) (Chapadão do Sul, MS, 2008). 

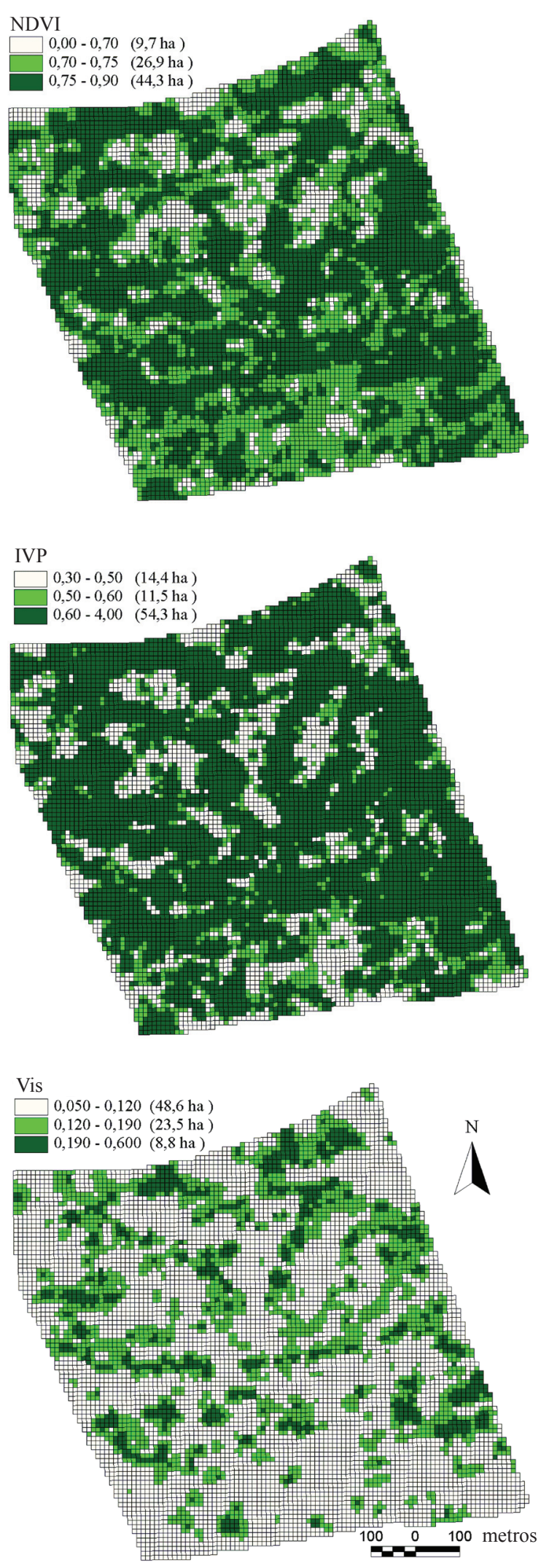

Figura 2. Mapas da distribuição espacial do NDVI, reflectância no infravermelho próximo (IVP) e no visível (Vis), na área 2 (Talhão 2) (Chapadão do Sul, MS, 2008). onde os sintomas de infestação de percevejos estavam mais evidentes, visualmente, ou seja, plantas menos desenvolvidas e amareladas.

Os resultados aqui obtidos permitem identificar regiões na lavoura que apresentam maior incidência de pragas de solo. Estas regiões, consideradas regiões homogêneas de manejo específico, podem ser controladas quimicamente, pela aplicação de inseticidas a taxas variáveis, proporcionando, desta forma, como afirmaram Corá et al. (2004), um manejo mais eficiente e econômico da cultura.

\section{CONCLUSÕES}

1. Os valores do NDVI foram semelhantes na área $1 \mathrm{e}$ área 2, com coeficientes de variação considerados baixos. Os índices de reflectância no infravermelho próximo e no visível apresentaram coeficiente de variação elevado, na área 2.

2. Na lavoura com maior estresse fisiológico, devido ao ataque do percevejo castanho, ocorreu maior variabilidade espacial do NDVI e da reflectância espectral da vegetação, na região do IVP e do visível, em relação à lavoura sadia. A identificação de regiões na lavoura que apresentam maior incidência de pragas permite que estas sejam tratadas como regiões homogêneas de manejo específico.

\section{REFERÊNCIAS}

ANDRADE, A. R. S. et al. Variabilidade espacial da densidade do solo sob manejo da irrigação. Ciência e Agrotecnologia, Lavras, v. 29, n. 2, p. 322-329, 2005.

BARET, F.; GUYOT, G. Potentials and limits of vegetation indices for LAI and APAR assessment. Remote Sensing of Environment, New York, v. 35, n. 1, p. 161-173, 1991.

BOOLTINK, H. W. G.; VERHAGEN, J. Integration of remote sensing, modeling and field measurements towards an operational decision support system for precision agriculture. In: EUROPEAN CONFERENCE ON PRECISION AGRICULTURE, 1., 1997, Warwick. Proceedings... Oxford: Bioss Scientific, 1997. p. 921-929. 1 CD-ROM.

CAMBARDELLA, C. A. et al. Field-scale variability of soil properties in central Iowa soils. Soil Science Society of America Journal, Madison, v. 58, n. 5, p. 1501-1511, 1994.

CARVALHO, J. R. P.; SILVEIRA, P. M.; VIEIRA, S. R. Geoestatística na determinação da variabilidade espacial de características químicas do solo sob diferentes preparos. 
Pesquisa Agropecuária Brasileira, Brasília, DF, v. 37, n. 8, p. 1151-1159, 2002.

CORÁ, J. E. et al. Variabilidade espacial de atributos do solo para adoção do sistema de agricultura de precisão na cultura de cana-de-açúcar. Revista Brasileira de Ciência do Solo, Viçosa, v. 28, n. 6, p. 1013-1021, 2004.

FARIA, G. E. et al. Soil fertility, organic carbon and fractions of the organic matter at different distances from eucalyptus stumps. Revista Brasileira de Ciência do Solo, Viçosa, v. 33, n. 3, p. 571-579, 2009.

GOEL, P. K. et al. Potential of airborne hyperspectral remote sensing to detect nitrogen deficiency and weed infestation in corn. Computers and Electronics in Agriculture, Oxford, v. 38, n. 2, p. 99-124, 2003.

ISAAKS, E. H.; SRIVASTAVA, R. M. An introduction to applied geostatistics. New York: Oxford University Press, 1989.

JULSING, M. K. et al. Combinatorial biosynthesis of medicinal plant secondary metabolites. Biomolecular Engeniering, New York, v. 23, n. 6, p. 265-79, 2006.

LAWRENCE, P. R. et al. Design and testing of a global positioning system-based radiometer for precision mapping of pearl millet total dry matter in the Sahel. Agronomy Journal, Madison, v. 92, n. 6, p. 1086-1095, 2000.

LI, H. et al. Spectral reflectance characteristics of cotton related to soil water and topography variability. In: INTERNATIONAL CONFERENCE ON PRECISION AGRICULTURE, 5., 2000, Minneapolis. Proceedings... Madison: ASA/CSSA/SSSA, 2000. 1 CD-ROM.

MA, B. L. et al. Early prediction of soybean yield from canopy reflectance measurements. Agronomy Journal, Madison, v. 93, n. 6, p. 1227-1234, 2001.

MARUR, C. J.; RUANO, O. A reference system for determination of developmental stages of upland cotton. Revista de Oleaginosas e Fibrosas, Campina Grande, v. 5, n. 2, p. 313-317, 2001.

MENDES, A. M. S.; FONTES, R. L. F.; OLIVEIRA, M. Variabilidade espacial da textura de dois solos do Deserto Salino, no Estado do Rio Grande do Norte. Ciência Agronômica, Fortaleza, v. 39, n. 1, p. 19-27, 2008.

MOREIRA, M. A. Fundamentos do sensoriamento remoto e metodologia de aplicação. São José dos Campos: INPE, 2001.

MOTOMIYA, A. V. A; CORÁ, J. E.; PEREIRA, G. T. Uso da krigagem indicatriz na avaliação de indicadores de fertilidade do solo. Revista Brasileira de Ciência do Solo, Viçosa, v. 30, n. 3, p. 485-496, 2006.
MOTOMIYA, A. V. A.; MOLIN, J. P.; CHIAVEGATO, E. D. Utilização de sensor óptico ativo para detectar deficiência foliar de nitrogênio em algodoeiro. Revista Brasileira de Engenharia Agrícola e Ambiental, Campina Grande, v. 13, n. 2, p. 137-145, 2009.

MULLA, D. J.; SCHEPERS, J. S. Key process and properties for site-specific soil and crop management. In: PIERCE, F. J.; SADLER, E. J. (Eds.). The site-specific management for agricultural systems. Madison: ASA/ CSSA/SSSA, 1997. p. 1-18.

POZONI, F. J.; SHIMABUKURO, Y. E. Sensoriamento remoto no estudo da vegetação. São José dos Campos: Parêntese, 2009.

ROBERTSON, G. P. GS+: Geostatistics for the environmental sciences. Versão 5.03 Beta. Plainwell: Gamma Design Software, 1998.

SILVA, F. M. et al. Variabilidade espacial de atributos químicos e produtividade da cultura do café em duas safras agrícolas. Ciência e Agrotecnologia, Lavras, v. 32, n. 1, p. 231-241, 2008.

SOLIE, J. B. et al. Real-time sensing and $\mathrm{N}$ fertilization with a field scale GreenSeeker applicator. In: INTERNATIONAL CONFERENCE ON PRECISION AGRICUlture, 6., 2002, Madison. Proceedings... Madison: ASA/CSSA/SSSA, 2002. 1 CD-ROM.

SOUZA, Z. M. et al. Influência da variabilidade espacial de atributos químicos de um latossolo na aplicação de insumos para cultura de cana-de-acúcar. Ciência e Agrotecnologia, Lavras, v. 1, n. 2, p. 71-377, 2007.

TUMBO, S. D.; WAGNER, D. G.; HEINEMANN, P. H. Hyperspectral characteristics of corn plants under different chlorophyll levels. Transactions of the ASAE, St. Joseph, v. 45 , n. 3, p. 815-823, 2002.

VIEIRA, S. R. Geoestatística em estudos de variabilidade espacial do solo. In: NOVAIS, R. F.; ÁLVARES, V. H.; SCHAEFFER, C. E. G. R. Tópicos em ciência do solo. Viçosa: Sociedade Brasileira de Ciência do Solo, 2000. p. 1-54.

VIEIRA, V. A. S.; MELLO, C. R.; LIMA, J. M. Variabilidade espacial de atributos físicos do solo em uma microbacia hidrográfica. Ciência e Agrotecnologia, Lavras, v. 31, n. 5, p. 1477-1485, 2007.

WILDING, L. P.; DREES, L. R. Spatial variability and pedology. In: WILDING, L. P.; SMECK, N. E.; HALL, G. F. Pedogenesis and soil taxonomy: concepts and interactions. New York: Elsevier, 1983. p. 83-116. 